\title{
Evaluation the relationship of left ventricular global longitudinal strain and laboratory parameters in discharged patients with COVID-19: a follow-up study
}

\author{
Derya Baykiz ${ }^{1}$. Elif Ayduk Govdeli ${ }^{1}$ Pelin Karaca Ozer ${ }^{1}$. Ekrem Bilal Karaayvaz ${ }^{1}$ Yunus Catma ${ }^{2}$. \\ Alpay Medetalibeyoglu ${ }^{2}$ Atahan Cagatay ${ }^{3} \cdot$ Berrin Umman $^{1} \cdot$ Tufan Tukek $^{2} \cdot$ Zehra Bugra $^{1}$
}

Received: 15 January 2021 / Accepted: 19 March 2021 / Published online: 7 April 2021

(c) The Author(s), under exclusive licence to Springer Nature B.V. 2021

\begin{abstract}
Background: The novel coronavirus infection (COVID-19) disease has spread rapidly and posed a great threat to global public health. The laboratory parameters and clinical outcomes of the disease in discharged patients remain unknown. In this study, we aimed to investigate the laboratory and echocardiographic findings of patients with COVID-19 after discharge and the relation between left ventricular global longitudinal strain (LVGLS) and inflammatory parameters in discharged patients. Methods: A total of 75 patients recovering from COVID-19 as the study group were prospectively recruited from the COVID-19 outpatient clinic for their follow-up visits at a median 6 months after discharge. Patients were classified into groups according to pneumonia severity and impairment in LVGLS. Laboratory findings of patients both at admission and after discharge were evaluated and the relation with pneumonia severity at admission and LVGLS after discharge were analyzed. Results: Serum ferritin, lactate dehydrogenase (LDH) and prohormone B-type natriuretic peptide (pro-BNP) levels after discharge were significantly higher in the study group than the control group $(n=44)$. Ferritin was found to be related to pneumonia severity. Serum ferritin and LDH values after discharge were significantly higher in patients with impaired LVGLS than those with preserved. There was a significant correlation between LVGLS, serum ferritin and LDH values after discharge $(\mathrm{r}=-0.252, p=0.012 ; \mathrm{r}=-0.268, p=0.005$, respectively). Conclusions: Clinicians should pay close attention to the serum ferritin and LDH levels in discharged patients for predicting the severity of COVID-19 disease and early identification of subclinical left ventricular myocardial dysfunction.
\end{abstract}

Keywords COVID-19 $\cdot$ Left ventricular global longitudinal strain $\cdot$ Ferritin $\cdot$ Lactate dehydrogenase $\cdot$ Inflammation

\section{Introduction}

The new severe acute respiratory syndrome coronavirus 2 (SARS-CoV-2), which is the etiological agent of novel coronavirus infection (COVID-19) disease caused a worldwide pandemic. The first known COVID-19 case appeared in Wuhan, China, in Dec 2019, then in March 2020, COVID-19

Derya Baykiz

derya.baykiz@hotmail.com; drderyabaykiz@gmail.com

1 Department of Cardiology, Istanbul Faculty of Medicine, Istanbul University, Istanbul 34093, Turkey

2 Department of Internal Medicine, Istanbul Faculty of Medicine, Istanbul University, Istanbul 34093, Turkey

3 Department of Infection Diseases, Istanbul Faculty of Medicine, Istanbul University, Istanbul 34093, Turkey was declared a pandemic by the World Health Organization (WHO). It is still spreading rapidly around the world and threatening global health [1]. COVID-19 infection may exhibit several clinical manifestations varying from mild respiratory illness to severe pneumonia and acute respiratory distress syndrome (ARDS). The most common symptoms seen in patients with novel COVID-19 pneumonia are fever, cough, dyspnea and fatigue [2].

In COVID-19 infection, monocyte and macrophage systems are activated by rapid viral replication of SARS-CoV-2 and release cytokines and chemokines. The release of excessive pro-inflammatory cytokines and chemokines triggers inflammatory responses and leads to cytokine storms. Due to inflammatory cytokine storm, the disease progresses to ARDS, being generally fatal and it also causes multiorgan failure. 
Many studies have shown that inflammatory responses play a pivotal role in the severity and prognosis of COVID19 disease [3]. Several inflammatory markers have been found to be related to disease severity and mortality in hospitalized patients. These inflammatory markers such as procalcitonin (PCT), C-reactive protein (CRP), lactate dehydrogenase (LDH), serum ferritin, and interleukin-6 (IL-6) have been observed to be significantly associated with the development of severe COVID-19 disease [4, 5]. Additionally, lymphocytopenia is common in COVID-19 infection and thought to be associated with disease severity and mortality [6].

Although COVID-19 affects multiple organ systems, the involvement of the cardiovascular system is of high importance to predict morbidity and mortality [2]. Prior studies have confirmed that COVID-19 generates acute myocardial injury and consequently cardiovascular complications [7]. The mechanism of acute myocardial injury in COVID-19 is not clear, however it can be explained by several mechanisms such as ischaemic myocardial injury, myocarditis, microvascular damage, hypoxemia, arrhythmias, cytokinerelated injury, or even stress cardiomyopathy $[7,8]$.

To show the degrees of myocardial injury, elevetad cardiac biomarkers and/or abnormalities on transthoracic echocardiography (TTE) can be used. Although TTE is the initial imaging modality to detect myocardial damage of COVID19, speckle-tracking echocardiography (STE) which tracks unique speckle pathways during the cardiac cycle provides an objective measurement of myocardial deformation and strain values. Left ventricular global longitudinal strain (LVGLS) measured by STE, has been shown to have prognostic implications independent of $\mathrm{LV}$ ejection fraction (LVEF) in many cardiovascular diseases [9].

LVGLS imaging is recommended in clinical practice to identify subclinical myocardial dysfunction. Reduced LVGLS can identify LV dysfunction earlier than conventional methods [10]. In contrast to preserved or normal LVEF, longitudinal strains are reduced in early stages of the cardiomyopathies. Subclinical reduction in LV function identified by GLS may precede irreversible LV myocardial dysfunction and heart failure [11]. This condition is associated with increased risk of ventricular arrhythmias, cardiovascular morbidity and mortality.

We consider that the evaluation of the inflammatory markers in COVID-19 course is critically important in both diagnosis and follow-up. Therefore, in this study, we aimed to investigate the laboratory examination of patients recovered from COVID-19 both at hospital admission and after discharge and mainly analyzed the association of the inflammatory markers with the severity of pneumonia at admission and LVGLS after discharge. To the best of our knowledge, in the literature, there is no study investigating this relationship in discharged patients from COVID-19.

\section{Methods}

\section{Study population}

A total of 75 patients diagnosed with COVID-19 via positive result of real time reverse-transcription polymerase chain reaction (RT-PCR) for SARS-CoV-2 RNA between April 2020 and June 2020 were prospectively recruited to the study from our outpatient clinic. A total of 44 individuals matching the study group in age, gender and comorbidities were included as the control group. Written informed consent was obtained from all patients before the enrollment. The study complies with the Declaration of Helsinki, and this single-center, prospective, observational study protocol was approved by the local Ethics Committee of Istanbul University (Decision no: 2020/09/1185).

Patients with acute coronary events within 3 months, coronary artery disease, severe valvular heart disease, patients with an EF of $<50 \%$, stage 2-3 hypertension, uncontrolled diabetes mellitus (HBA1c $\geq 8$ ), chronic renal failure (estimated GFR $<30 \mathrm{~mL} / \mathrm{min} / 1.73 \mathrm{~m}^{2}$ ), severe chronic lung or chronic liver disease, chronic inflammatory diseases, rheumatic diseases, the history of myocarditis, malignancy, patients younger than 18 years of age, thyroid disorder and poor echogenicity were excluded from the study.

\section{Study protocol}

Demographic, laboratory and clinical data at hospital admission were obtained from the hospital electronic medical records and carefully reviewed. Additionally, the control laboratory findings of the patients were recorded and a detailed transthoracic echocardiographic examination was performed in all patients when they come to have their follow-up visits to our COVID-19 outpatient clinic at a median 6 months after discharge.

Complete blood count, including total white blood cell (WBC) counts, neutrophil, and lymphocyte counts, neutrophil and lymphocyte percentages, and biochemical analyses such as renal and liver function parameters, CRP, D-dimer, fibrinogen, PCT, ferritin, LDH and prohormone B-type natriuretic peptide (pro-BNP) both at hospital admission and after discharge were determined for all patients. Besides, neutrophil-to-lymphocyte ratio (NLR) which is a composite index of systemic inflammation was calculated. The hemogram data of the patients were studied with the Beckman Coulter L780 device and the biochemical tests with the Roche Cobas C501-C601 device.

\section{TTE and STE}

On the same day, a detailed transthoracic echocardiographic examination and 2D-STE with an iE33xMATRIX ultrasound 
system (Philips Medical Systems, Andover, Massachusetts) using an X5-1 (1-5 MHz) transducer were performed in all patients by two experienced cardiologists blinded to the study groups. Conventional echocardiographic measurements were performed by the recommendations of the American Society of Echocardiography [12]. Echocardiographic images were obtained in the parasternal and apical views. LVEF, LV end-diastolic volume (EDV), LV end-systolic volume (ESV) were measured using the modified Simpson's biplane method [13]. Left atrial volume was measured using the biplane method in four and two apical chamber views and indexed to body surface area (BSA).

LV diastolic function was assessed by the peak early diastolic filling (E wave) velocity to the late diastolic filling (A wave) velocity as the E/A ratio and transmitral $\mathrm{E}$ to the mean of LV septal and lateral early diastolic tissue velocities (mean e') as E/ e' ratio. Right ventricular (RV) diameter was determined from the apical four chamber view. Tricuspid annular plane systolic excursion (TAPSE) was measured on M-mode. Systolic pulmonary artery pressure (sPAP) was calculated by tricuspid regurgitation peak velocity.

\section{D-STE}

LVGLS was measured by 2D-STE to determine myocardial deformation. Measurements of LVGLS were performed offline using commercially available software (QLAb) program. The endocardial borders were automatically traced by the software program at the end of systole by aortic valve closure. If required, manual adjustments were made by the physician.

The apical four-three and two chamber views were used for LVGLS analysis. The mean GLS was measured by averaging the peak GLS values of apical four-,three-, and two- chamber images. LVGLS analysis was made by a single experienced cardiologist (EAG). The cut off value of impaired LVGLS was accepted as $-16 \%$ as described in previous studies $[14,15]$. An equal or lower than $-16 \%$ of LVGLS was determined as impaired LVGLS.

\section{Grouping}

The study population included 75 patients who recovered from COVID-19 infection as the study group and 44 patients who had no COVID-19 infection as the control group. The study group was categorized into 3 groups as those with mild pneumonia, those with severe pneumonia and those without pulmonary involvement based on chest computed tomography (CT) reports at admission.

All patients had an equal or greater than $50 \%$ of LVEF. Besides, the study group was classified into 2 groups based on LVGLS values after discharge which were determined to be impaired or preserved. The laboratory findings of patients both at admission and after discharge were assessed between 2 groups.

\section{Statistical methods}

All statistical tests were conducted using the Statistical Package for the Social Sciences 26.0 for Windows (SPSS Inc., Chicago, IL, USA). The Kolmogorov-Smirnov test was used to analyze the normality of the data. Continuous data are expressed as mean \pm standard deviation (SD), and categorical data are expressed as percentages. A chi-square or Fisher's exact test was used to assess the differences in categorical variables between the groups. A Student's t-test or the Mann-Whitney U test was used to compare unpaired samples as needed. The relationships among the parameters were assessed using Pearson's or Spearman's correlation analysis according to the normality of the data. The primary analysis used ANOVA to compare all reported data for parametric variables, whereas the Kruskal-Wallis test was used for comparison among non-parametric variables between the groups. We used Tukey's or Tamhane's post hoc analysis test for significant deviations showed by ANOVA and used Dunn's test for non-parametric pairwise multiple comparisons procedure by the Kruskal-Wallis. A $p$-value of 0.017 adjusted by the Bonferroni method was used in pairwise comparisons of categorical variables. The receiver operating characteristic (ROC) curve, sensitivity, specificity and area under the curve (AUC) were calculated to evaluate the values of biochemical markers in predicting the impairment of LVGLS. Significance was assumed at a two-sided $p<0.05$.

\section{Results}

\section{Clinical characteristics and laboratory findings}

A total of 119 patients were enrolled in the present study. Of 119 patients, 44 patients were the control group and 75 patients consisted the study group (Fig. 1). The demographic, clinical data, echocardiographic and laboratory findings of the subjects are presented in Table 1 . The mean age of the study group was $49.6 \pm 13.6$ years and the mean age of the control group was $44.9 \pm 12.7$ years. Among 119 patients, $50(42 \%)$ were male and $69(58 \%)$ were female. There was no statistically significant difference between the groups in terms of age, gender, current smoking, diabetes mellitus, hypertension, resting heart rate and body mass index.

In laboratory findings after discharge; serum ferritin, LDH and pro-BNP levels were significantly higher in the study group compared to the control group. There was no statistical difference between the groups in terms of CRP, 


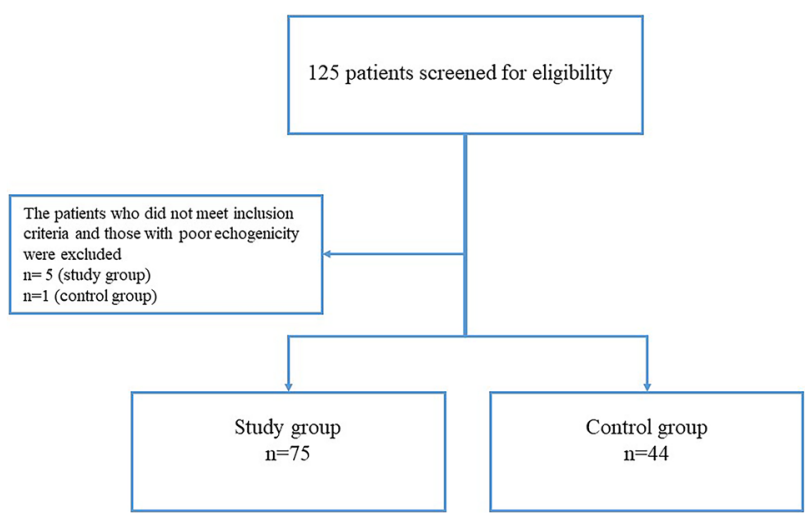

Fig. 1 Flow chart of the study design

D-dimer, fibrinogen, NLR, hematologic and other biochemical parameters.

Baseline and clinical characteristics, the treatment received, echocardiographic parameters and laboratory findings of the study population according to pneumonia severity were described in Table $2 a, b$. Serum ferritin values both at admission and after discharge were significantly higher in patients with COVID-19 pneumonia compared to those without pulmonary involvement. Serum LDH levels in both visits tended to be higher in the severe pneumonia group but this finding did not reach statistical significance. Lymphocyte, WBC and platelet counts after discharge were significantly different among the groups. Other inflammatory and hematologic parameters both at admission and after discharge showed no difference between the groups.

\section{Echocardiographic characteristics}

Conventional echocardiography parameters and LVGLS values were compared between the study and control group in follow-up visit (Table 1).Compared with the control group; LVEF values, LV end-diastole diameter (LVEDD), right atrial (RA) diameter, E/e' ratio and left atrial volume index (LAVI) showed no significant difference in the study group. However, LVEDV, LVESV, left atrial (LA) diameter, RV diameter, TAPSE and SPAP were significantly higher and LVGLS values and E/A ratio were significantly lower in the study group compared to the control group (Fig. 2). The LVGLS values of the control and study groups were $-18.3 \pm 2.3 \%$, and $-16.7 \pm 3.7 \%$, respectively $(p=0.01)$.

In addition, according to the groups of pneumonia severity at admission; LA diameter, E/A ratio, TAPSE, sPAP and LVGLS values showed statistically significant difference among the groups in follow-up (Table 2).

Table 3 shows the comparison of the laboratory findings between the patients with preserved LVGLS $>-16 \%$ and impaired LVGLS $\leq-16 \%$. Serum ferritin, LDH, pro-BNP and hemoglobin values after discharge were significantly higher in patients with impaired LVGLS compared to those with preserved (Table 3, Fig. 3).Platelet counts were found to be lower in patients with impaired LVGLS. There was no significant association between LVGLS and the other laboratory parameters both at admission and after discharge (Table 3).

In correlation analysis; LVGLS was negatively correlated with age, pneumonia severity, ferritin and CRP levels at hospital admission $(\mathrm{r}=-0.270, p=0.004 ; \mathrm{r}=-0.352, p<0.001$; $\mathrm{r}=-0.300, p=0.01$; and $\mathrm{r}=-0.280, p=0.01$, respectively). There was a significant correlation between LVGLS values, serum ferritin and LDH levels after discharge $(r=-0.252$, $p=0.012 ; \mathrm{r}=-0.268, p=0.005$, respectively) (Table 4, Fig. 4).

According to ROC analysis; serum ferritin values at hospital admission predict impaired LVGLS with $67.9 \%$ sensitivity and $67.4 \%$ specificity with a cutoff of $113.05 \mathrm{ng} / \mathrm{ml}$ ( $p=0.004$, AUC $0.698,95 \%$ CI 0.572-0.824). Serum ferritin values after discharge predict impaired LVGLS with $54.8 \%$ sensitivity and $67.9 \%$ specificity with a cutoff of $56 \mathrm{ng} / \mathrm{ml}$ ( $p=0.005$, AUC $0.668,95 \%$ CI $0.562-0.773$ ) (Fig. 5).

A cutoff value of $22.42 \mathrm{mg} / \mathrm{l}$ of serum CRP levels at admission resulted in a $56.7 \%$ sensitivity and $79.2 \%$ specificity for predicting impaired LVGLS $(p=0.006$, AUC 0.684 , 95\% CI 0.560-0.808).

Serum LDH values after discharge predict impaired LVGLS with $68.2 \%$ sensitivity and.

$66.2 \%$ specificity with a cutoff of $188.5 \mathrm{U} / 1(p=0.001$, AUC 0.693, 95\% CI 0.594-0.792).

\section{Discussion}

In this study, we analyzed the laboratory examination of COVID-19 patients, especially hematological and inflammatory parameters both at admission and after discharge and evaluated the relationship with LVGLS in follow-up. The remarkable findings of our study are as follows:

(i) Among the inflammatory markers, only serum ferritin levels both at hospital admission and after discharge were found to be significantly associated with the severity of pneumonia.

(ii) LVGLS values after discharge were significantly lower in the group with severe pneumonia than those without pulmonary involvement (see Table2).

(iii) As the most interesting findings of our study, the patients recovering from the COVID-19 infection who had higher serum ferritin and LDH levels in control laboratory analysis after discharge demonstrated reduced LVGLS values in follow-up visits. We consider that these patients may benefit from 
Table 1 Baseline, clinical characteristics, laboratory and echocardiographic findings of study and control groups

\begin{tabular}{|c|c|c|c|c|}
\hline & Total patients $(n=119)$ & COVI D-19 $(n=75)$ & Control $(n=44)$ & $p$-value \\
\hline Age (year) & $48 \pm 13.4$ & $49.6 \pm 13.6$ & $44.9 \pm 12.7$ & 0.072 \\
\hline Gender, Male, $n(\%)$ & $50(42 \%)$ & $36(48 \%)$ & $14(31.8 \%)$ & 0.084 \\
\hline Female, $n(\%)$ & $69(58 \%)$ & $39(52 \%)$ & $30(68.2 \%)$ & \\
\hline HR (bpm) & $79.5 \pm 13.8$ & $80.9 \pm 13.6$ & $76.7 \pm 14$ & 0.146 \\
\hline BMI $\left(\mathrm{kg} / \mathrm{m}^{2}\right)$ & $28 \pm 5$ & $28.5 \pm 4.7$ & $27.2 \pm 5.4$ & 0.162 \\
\hline $\mathrm{HT}, n(\%)$ & $33(27.7 \%)$ & $23(30.7 \%)$ & $10(22.7 \%)$ & 0.350 \\
\hline $\mathrm{DM}, n(\%)$ & $18(15.1 \%)$ & $13(17.3 \%)$ & $5(11.4 \%)$ & 0.380 \\
\hline Smoking, $n(\%)$ & $33(27.7 \%)$ & $17(22.7 \%)$ & $16(36.4 \%)$ & 0.107 \\
\hline \multicolumn{5}{|l|}{ Laboratory findings after discharge } \\
\hline Ferritin $(\mathrm{ng} / \mathrm{ml})$ & $49.8(3-1010)$ & $53.5(7-1010)$ & $32(3-212)$ & $0.034^{*}$ \\
\hline Fibrinogen (mg/dl) & $333.5(180-648)$ & $328(180-511)$ & $344.5(287-648)$ & 0.105 \\
\hline $\mathrm{CRP}(\mathrm{mg} / \mathrm{l})$ & $2(0-38.8)$ & $2.3(0.1-38.8)$ & $2(0-24)$ & 0.747 \\
\hline D-dimer $(\mu \mathrm{g} / \mathrm{L})$ & $340(170-1910)$ & $340(170-1910)$ & $470(210-1330)$ & 0.343 \\
\hline $\mathrm{Hgb}(\mathrm{gr} / \mathrm{dl})$ & $13.2(8.5-17)$ & $13.2(8.5-16.4)$ & $13(11-17)$ & 0.097 \\
\hline $\operatorname{RBC}\left(10^{6} / \mu \mathrm{l}\right)$ & $4.7(0.2-5.7)$ & $4.7(0.2-5.7)$ & $4.6(3.6-5.7)$ & 0.579 \\
\hline Hematocrit (\%) & $40(19-49.2)$ & $40.8(19-48.3)$ & $40(33.2-49.2)$ & 0.871 \\
\hline Neutrophil/Lymphocyte Ratio & $1.8(0.6-7.6)$ & $1.8(0.6-7.6)$ & $1.9(0.6-3.8)$ & 0.969 \\
\hline Neutrophil $\left(10^{3} / \mu \mathrm{l}\right)$ & $3.7(1.4-16.6)$ & $3.5(1.4-16.6)$ & $3.9(1.6-7.8)$ & 0.205 \\
\hline Lymphocyte $\left(10^{3} / \mu \mathrm{l}\right)$ & $2.2(0.5-6.9)$ & $2.1(0.5-4.1)$ & $2.4(1.1-6.9)$ & 0.105 \\
\hline $\operatorname{WBC}\left(10^{3} / \mu \mathrm{l}\right)$ & $6.7(2.4-19.6)$ & $6.1(2.4-19.6)$ & $7(4.4-13.5)$ & 0.149 \\
\hline Lymphocyte \% & $32.9 \pm 7.7$ & $32.7 \pm 7.7$ & $33.2 \pm 7.8$ & 0.721 \\
\hline Neutrophil \% & $57 \pm 8.1$ & $57 \pm 8.3$ & $57.1 \pm 7.9$ & 0.925 \\
\hline Platelet $\left(10^{3} / \mu \mathrm{l}\right)$ & $239.5(39.6-409)$ & $236(39.6-409)$ & $241(179-373)$ & 0.305 \\
\hline Pro-BNP (pg/ml) & $43(4-1258)$ & $57(5-1258)$ & $34(4-44)$ & $0.005^{*}$ \\
\hline $\operatorname{AST}(\mathrm{U} / \mathrm{l})$ & $18.8(9.3-237)$ & $19.1(10.4-237)$ & $17.6(9.3-82.9)$ & 0.126 \\
\hline $\operatorname{ALT}(\mathrm{U} / \mathrm{l})$ & $18.4(4.9-205)$ & $19.2(4.9-205)$ & $18.2(8-80.3)$ & 0.770 \\
\hline BUN (mg/dl) & $13.2(5.9-44.3)$ & $13.8(6-37.1)$ & $11.9(5.9-44.3)$ & 0.230 \\
\hline Creatinine (mg/dl) & $0.8(0.4-1.7)$ & $0.8(0.4-1.7)$ & $0.8(0.5-1.2)$ & 0.372 \\
\hline LDH (U/l) & $189.4 \pm 39.6$ & $195.4 \pm 34.3$ & $176.3 \pm 47.3$ & $0.019^{*}$ \\
\hline Uric acid (mg/dl) & $5.2 \pm 1.6$ & $5.3 \pm 1.7$ & $4.9 \pm 1.6$ & 0.175 \\
\hline Fasting Plasma Glucose (mg/dl) & $92.5(69-268)$ & $94(69-268)$ & $92.5(69-159)$ & 0.883 \\
\hline \multicolumn{5}{|l|}{ Echocardiographic findings } \\
\hline LVEDV (ml) & $135.8 \pm 24.4$ & $138.9 \pm 24.1$ & $129.9 \pm 24.2$ & $0.050^{*}$ \\
\hline LVESV (ml) & $53.5 \pm 12.1$ & $54.8 \pm 12.1$ & $51.2 \pm 11.9$ & $0.036^{*}$ \\
\hline $\operatorname{LVEF}(\%)$ & $64.8 \pm 4.6$ & $64.8 \pm 4.9$ & $65 \pm 3.9$ & 0.841 \\
\hline LVEDD (mm) & $45 \pm 3.9$ & $45.5 \pm 3.9$ & $44.1 \pm 4$ & 0.055 \\
\hline $\mathrm{LA}(\mathrm{mm})$ & $34.7 \pm 4.5$ & $36 \pm 4.3$ & $32.4 \pm 4.1$ & $<0.001^{*}$ \\
\hline $\mathrm{RV}(\mathrm{mm})$ & $26.6 \pm 2.4$ & $26.9 \pm 2.1$ & $25.9 \pm 2.8$ & $0.020^{*}$ \\
\hline $\mathrm{RA}(\mathrm{mm})$ & $31.3 \pm 3.1$ & $31.6 \pm 3.2$ & $30.8 \pm 2.8$ & 0.207 \\
\hline $\mathrm{E} / \mathrm{A}$ ratio & $1 \pm 0.4$ & $0.9 \pm 0.3$ & $1.2 \pm 0.4$ & $<0.001^{*}$ \\
\hline E/e' ratio & $8.8 \pm 3$ & $8.9 \pm 3.2$ & $8.5 \pm 2.6$ & 0.782 \\
\hline LAVI $\left(\mathrm{ml} / \mathrm{m}^{2}\right)$ & $18.6 \pm 6.3$ & $18.8 \pm 6.6$ & $18.3 \pm 5.9$ & 0.586 \\
\hline TAPSE (mm) & $21.9 \pm 3.5$ & $21.5 \pm 3.5$ & $22.9 \pm 3.4$ & $0.046^{*}$ \\
\hline sPAP (mmHg) & $22.8 \pm 6.3$ & $26 \pm 5.1$ & $17.8 \pm 4.5$ & $<0.001^{*}$ \\
\hline LVGLS (\%) & $-17.3 \pm 3.4$ & $-16.7 \pm 3.7$ & $-18.3 \pm 2.3$ & $0.010^{*}$ \\
\hline
\end{tabular}

${ }^{*} P<0.05$ Between control group and study group. Abbreviations: $H R$ heart rate, $B M I$ body mass index, $H T$ hypertension, $D M$ diabetes mellitus, $C R P$ C reactive protein, $H g b$ hemoglobin, $R B C$ red blood cell, $W B C$ white blood cell, Pro- $B N P$ prohormone B-type natriuretic peptide, $A S T$ aspartate transaminase, $A L T$ alanine transaminase, $B U N$ blood urea nitrogen, $L D H$ lactate dehydrogenase, $L V E D V$ left ventricular end-diastolic volume, $L V E S V$ left ventricular end sistolic volume, $L V E F$ left ventricular ejection fraction, $L V E D D$ left ventricular end diastolic diameter, $L A$ left atrium, $R V$ right ventricle, $R A$ right atrium, $L A V I$ left atrium volume index, TAPSE tricuspid annular plane systolic excursion, $s P A P$ systolic pulmonary artery pressure, $L V G L S$ left ventricular global longitudinal strain 
Table 2 a: Demographic and clinical data, treatment received and echocardiographic findings of the groups. b: Comparison of laboratory parameters both at admission and after discharge between the groups according to pneumonia severity

\begin{tabular}{|c|c|c|c|c|c|}
\hline (a) & Control $(n=44)$ & $\begin{array}{l}\text { Without pulmonary } \\
\text { involvement }(n=34)\end{array}$ & Mild pneumonia $(n=30)$ & Severe pneumonia $(n=11)$ & $p$-value \\
\hline Age (year) & $44.9 \pm 12.7$ & $47.8 \pm 13.5$ & $52.2 \pm 12.3$ & $48 \pm 17.1$ & 0.161 \\
\hline Gender, Male, $n(\%)$ & $14(31.8 \%)$ & $14(41.2 \%)$ & $17(56.7 \%)$ & $5(45.5 \%)$ & 0.205 \\
\hline Female, $n(\%)$ & $30(68.2 \%)$ & $20(58.2 \%)$ & $13(43.3 \%)$ & $6(54.5 \%)$ & \\
\hline HR (bpm) & $76.7 \pm 14$ & $78.9 \pm 13$ & $82 \pm 11.8$ & $83.8 \pm 19.2$ & 0.319 \\
\hline BMI (kg/m2) & $27.2 \pm 5.4$ & $27.4 \pm 4.4$ & $29.5 \pm 4.1$ & $29.7 \pm 6.7$ & 0.180 \\
\hline $\mathrm{HT}, n(\%)$ & $10(22.7 \%)$ & $12(35.3 \%)$ & $7(23.3 \%)$ & $4(36.4 \%)$ & 0.528 \\
\hline $\mathrm{DM}, n(\%)$ & $5(11.4 \%)$ & $5(14.7 \%)$ & $6(20 \%)$ & $2(18.2 \%)$ & 0.771 \\
\hline Smoking, $n(\%)$ & $16(36.4 \%)$ & $8(23.5 \%)$ & $8(26.7 \%)$ & $1(9.1 \%)$ & 0.277 \\
\hline \multicolumn{6}{|l|}{ Treatment } \\
\hline Hydroxychloroquine, $\mathrm{n}(\%)$ & - & $34(100 \%)$ & $30(100 \%)$ & $11(100 \%)$ & - \\
\hline Azithromycin, $n(\%)$ & - & $11(32.4 \%)$ & $13(43.3 \%)$ & $6(54.5 \%)$ & 0.380 \\
\hline Favipiravir, $n(\%)$ & - & $5(14.7 \%)$ & $5(16.7 \%)$ & $4(36.4 \%)$ & 0.259 \\
\hline Steroid, $n(\%)$ & - & $0(0 \%)^{\mathrm{e}}$ & $1(3.3 \%)$ & $3(27.3 \%)^{\mathrm{e}}$ & $0.002^{*}$ \\
\hline Immune modulator, $n(\%)$ & - & $7(20.6 \%)$ & $7(23.3 \%)$ & $3(27.3 \%)$ & 0.894 \\
\hline Antibiotics, $n(\%)$ & - & $16(47.1 \%)$ & $11(36.7 \%)$ & $4(36.4 \%)$ & 0.657 \\
\hline Hospital stay (days) & - & $3.5(0-18)$ & $5.2(0-26)$ & $10.2(0-33)$ & 0.131 \\
\hline ICU admission, $n(\%)$ & - & $1(2.9 \%)$ & $1(3.3 \%)$ & $2(18.2 \%)$ & 0.121 \\
\hline NIMV/ intubation, $n(\%)$ & - & $3(8.8 \%)$ & $2(6.7 \%)$ & $2(18.2 \%)$ & 0.545 \\
\hline \multicolumn{6}{|l|}{ Echocardiographic findings } \\
\hline LVEDV (ml) & $129.9 \pm 24.2$ & $139.2 \pm 24$ & $139.7 \pm 26.6$ & $135.7 \pm 18.4$ & 0.266 \\
\hline LESV (ml) & $51.2 \pm 11.9$ & $54 \pm 14.1$ & $55.3 \pm 11.5$ & $55.6 \pm 6.7$ & 0.140 \\
\hline $\operatorname{LVEF}(\%)$ & $65 \pm 3.9$ & $65.2 \pm 5.1$ & $64.6 \pm 5.2$ & $63.6 \pm 3.1$ & 0.569 \\
\hline LVEDD (mm) & $44.1 \pm 4$ & $45.6 \pm 3.9$ & $45.6 \pm 4.2$ & $45.1 \pm 3$ & 0.282 \\
\hline $\mathrm{LA}(\mathrm{mm})$ & $32.4 \pm 4.1^{\mathrm{a}, \mathrm{b}, \mathrm{c}}$ & $35.3 \pm 4.2^{\mathrm{a}}$ & $36.2 \pm 4.5^{\mathrm{b}}$ & $37.5 \pm 4.2^{\mathrm{c}}$ & $0.001^{*}$ \\
\hline $\mathrm{RV}(\mathrm{mm})$ & $25.9 \pm 2.8$ & $26.7 \pm 2.2$ & $27.4 \pm 2.1$ & $26.5 \pm 1.6$ & 0.070 \\
\hline $\mathrm{RA}(\mathrm{mm})$ & $30.8 \pm 2.8$ & $31.1 \pm 3.4$ & $32.1 \pm 3.3$ & $31.8 \pm 2.3$ & 0.398 \\
\hline E/A ratio & $1.2 \pm 0.4^{\mathrm{a}, \mathrm{b}, \mathrm{c}}$ & $1 \pm 0.3^{\mathrm{a}, \mathrm{d}}$ & $0.9 \pm 0.3^{b, d}$ & $0.9 \pm 0.3^{\mathrm{c}}$ & $<0.001^{*}$ \\
\hline E/e' ratio & $8.5 \pm 2.6$ & $9.1 \pm 3.8$ & $9 \pm 2.9$ & $8.4 \pm 1.4$ & 0.939 \\
\hline $\operatorname{LAVI}\left(\mathrm{ml} / \mathrm{m}^{2}\right)$ & $18.3 \pm 5.9$ & $20 \pm 7.6$ & $17.9 \pm 6.3$ & $17.6 \pm 2.2$ & 0.602 \\
\hline TAPSE (mm) & $22.9 \pm 3.4^{\mathrm{b}}$ & $21.9 \pm 3.4^{\mathrm{d}}$ & $20.1 \pm 2.9^{\mathrm{b}, \mathrm{d}, \mathrm{f}}$ & $23.6 \pm 4.2^{\mathrm{f}}$ & $0.008^{*}$ \\
\hline sPAP (mmHg) & $17.8 \pm 4.5^{\mathrm{a}, \mathrm{b}, \mathrm{c}}$ & $25.7 \pm 5.5^{\mathrm{a}}$ & $26.4 \pm 4.9^{\mathrm{b}}$ & $26 \pm 4.3^{c}$ & $<0.001^{*}$ \\
\hline LVGLS $(\%)$ & $-18.3 \pm 2.3^{\mathrm{b}, \mathrm{c}}$ & $-18.2 \pm 3.7^{\mathrm{d}, \mathrm{e}}$ & $-15.3 \pm 3.3^{\mathrm{b}, \mathrm{d}}$ & $-15.6 \pm 3.4^{\mathrm{c}, \mathrm{e}}$ & $<0.001^{*}$ \\
\hline (b) & Control $(n=44)$ & $\begin{array}{l}\text { Without pulmonary } \\
\text { involvement }(n=34)\end{array}$ & Mild pneumonia $(n=30)$ & Severe pneumonia $(n=11)$ & $\overline{p \text {-value }}$ \\
\hline
\end{tabular}

Laboratory findings after discharge

Ferritin (ng/ml)

Fibrinogen $(\mathrm{mg} / \mathrm{dl})$

CRP (mg/l)

D-dimer $(\mu \mathrm{g} / \mathrm{L})$

$\mathrm{Hgb}(\mathrm{gr} / \mathrm{dl})$

$\operatorname{RBC}\left(10^{6} / \mu \mathrm{l}\right)$

Hematocrit (\%)

Neutrophil/Lymphocyte Ratio

Neutrophil $\left(10^{3} / \mu \mathrm{l}\right)$

Lymphocyte $\left(10^{3} / \mu \mathrm{l}\right)$

WBC $\left(10^{3} / \mu \mathrm{l}\right)$

Lymphocyte \%

Neutrophil \%

$\begin{array}{lll}32(3-212)^{\mathrm{b}} & 33.5(7-359.5)^{\mathrm{d}} & 81.7(7.9-1010)^{\mathrm{b}, \mathrm{d}} \\ 344.5(287-648) & 298(218-511) & 337(180-472) \\ 2(0-24) & 1.4(0.1-17.4) & 3.5(0.4-23.7) \\ 470(210-1330) & 310(180-920) & 340(170-1910) \\ 13(11-17) & 13(8.5-15.9) & 13.3(8.8-16.2) \\ 4.6(3.6-5.7) & 4.6(2.8-5.7) & 4.9(0.2-5.6) \\ 40(33.2-49.2) & 39.2(25.8-47.6) & 42.2(19-48.3) \\ 1.9(0.6-3.8) & 1.9(1.1-5.5) & 1.7(0.7-7.6) \\ 3.9(1.6-7.8) & 3.5(1.4-7.7) & 3.300(1.9-16.6) \\ 2.4(1.1-6.9)^{\mathrm{a}} & 1.8(0.5-3.8)^{\mathrm{a}, \mathrm{e}} & 2.2(1.1-4.1)^{\mathrm{f}} \\ 7(4.4-13.5)^{\mathrm{a}} & 5.9(2.4-10.8)^{\mathrm{a}, \mathrm{e}} & 6(4.1-19.6) \\ 33.2 \pm 7.8 & 32 \pm 7.1 & 32.2 \pm 8.8 \\ 57.1 \pm 7.9 & 57.5 \pm 7.1 & 57.2 \pm 10\end{array}$

\begin{tabular}{ll}
$51.8(14-396.2)$ & $0.014^{*}$ \\
$347(302-374)$ & 0.074 \\
$2.3(0.8-38.8)$ & 0.184 \\
$390(270-780)$ & 0.353 \\
$13(11.4-16.4)$ & 0.345 \\
$4.6(3.9-5.3)$ & 0.360 \\
$40.9(33.4-44.8)$ & 0.438 \\
$1.4(0.6-2.8)$ & 0.348 \\
$3.7(2.5-8)$ & 0.540 \\
$2.9(1.9-4.1)^{\mathrm{e}, \mathrm{f}}$ & $0.002^{*}$ \\
$7.3(5.5-12)^{\mathrm{e}}$ & $0.025^{*}$ \\
$36 \pm 6$ & 0.851 \\
$54.7 \pm 7.5$ & 0.340 \\
\hline
\end{tabular}


Table 2 (continued)

\begin{tabular}{|c|c|c|c|c|c|}
\hline (b) & Control $(n=44)$ & $\begin{array}{l}\text { Without pulmonary } \\
\text { involvement }(n=34)\end{array}$ & Mild pneumonia $(n=30)$ & Severe pneumonia $(n=11)$ & $p$-value \\
\hline Platelet $\left(10^{3} / \mu \mathrm{l}\right)$ & $241(179-373)^{\mathrm{b}}$ & $260(155-409)^{\mathrm{d}}$ & $213(39.6-362)^{\mathrm{b}, \mathrm{d}}$ & $229(147-389)$ & $0.020^{*}$ \\
\hline Pro-BNP (pg/ml) & $34(4-44)^{\mathrm{a}, \mathrm{b}}$ & $55.5(5-1258)^{\mathrm{a}}$ & $60.4(5-621.5)^{\mathrm{b}}$ & $66.5(5-257.9)$ & $0.036^{*}$ \\
\hline LDH (U/l) & $176.3 \pm 47.3$ & $190.2 \pm 32$ & $199.3 \pm 25.4$ & $200.6 \pm 57.2$ & 0.087 \\
\hline \multicolumn{6}{|l|}{$\begin{array}{l}\text { Laboratory findings at hospital } \\
\text { admission }\end{array}$} \\
\hline Ferritin $(\mathrm{ng} / \mathrm{ml})$ & - & $158(22-1718)^{\mathrm{d}, \mathrm{e}}$ & $393.2(81.7-1654)^{\mathrm{d}}$ & $433.6(139.8-1396)^{\mathrm{e}}$ & $0.025^{*}$ \\
\hline Fibrinogen (mg/dl) & - & $468(293-714)$ & $504(204-747)$ & $322(301-587)$ & 0.268 \\
\hline CRP (mg/l) & - & $11.6(0.7-127.3)$ & $36(9.1-230.2)$ & $30.5(6.4-162.9)$ & 0.054 \\
\hline Procalcitonin (ng/ml) & - & $0(0-8.6)$ & $0.1(0-7.7)$ & $0.1(0-0.1)$ & 0.124 \\
\hline D-dimer $(\mu \mathrm{g} / \mathrm{L})$ & - & $450(270-7340)$ & $790(320-3440)$ & $1010(430-18.550)$ & 0.134 \\
\hline $\mathrm{Hgb}(\mathrm{gr} / \mathrm{dl})$ & - & $13.1(8.1-16.6)$ & $13(8.3-16.8)$ & $11.5(9.7-14.7)$ & 0.354 \\
\hline $\operatorname{RBC}\left(10^{6} / \mu \mathrm{l}\right)$ & - & $4.5(2.9-486)$ & $4.7(3-5.7)$ & $4(3.3-4.8)$ & 0.349 \\
\hline Hematocrit (\%) & - & $38.9(24.6-50.5)$ & $39.3(24.8-49.7)$ & $34.5(29.5-41.8)$ & 0.508 \\
\hline Neutrophil/Lymphocyte Ratio & - & $2.5(1-10.5)$ & $3.5(1.3-43.2)$ & $2.7(1.9-43.5)$ & 0.556 \\
\hline Neutrophil $\left(10^{3} / \mu \mathrm{l}\right)$ & - & $3.6(1.9-7.9)$ & $3.4(1.6-8.3)$ & $2.9(2.4-11.3)$ & 0.901 \\
\hline Lymphocyte $\left(10^{3} / \mu \mathrm{l}\right)$ & - & $1.5(0.2-2.7)$ & $1.2(0.8-3)$ & $0.9(0.2-5)$ & 0.391 \\
\hline $\operatorname{WBC}\left(10^{3} / \mu \mathrm{l}\right)$ & - & $6(3.2-9.8)$ & $4.6(3.9-9.9)$ & $6.2(3.6-15.9)$ & 0.606 \\
\hline Lymphocyte \% & - & $14.6 \pm 6$ & $21.6 \pm 8.4$ & $23.3 \pm 12.4$ & 0.650 \\
\hline Neutrophil \% & - & $63.1 \pm 11.7$ & $69.5 \pm 8.4$ & $68.9 \pm 14.5$ & 0.167 \\
\hline Platelet $\left(10^{3} / \mu \mathrm{l}\right)$ & - & $218(94-416)$ & $176(73-881)$ & $257(152-1100)$ & 0.450 \\
\hline Pro-BNP (pg/ml) & - & $80.9(7.4-711.1)$ & $56.3(12.7-793.1)$ & $81(19.2-564.9)$ & 0.654 \\
\hline LDH (U/l) & - & $281.7 \pm 101.7$ & $270.3 \pm 97.3$ & $431.3 \pm 245.9$ & 0.089 \\
\hline
\end{tabular}

Abbreviations: $H R$ heart rate, $B M I$ body mass index, $H T$ hypertension, $D M$ diabetes mellitus, $I C U$ intensive care unit, $N I M V$ non invasive mechanical ventilation, $L V E D V$ left ventricular end-diastolic volume, $L V E S V$ left ventricular end sistolic volume, $L V E F$ left ventricular ejection fraction, $L V E D D$ left ventricular end diastolic diameter, $L A$ left atrium, $R V$ right ventricle, $R A$ right atrium, $L A V I$ left atrium volume index, TAPSE tricuspid annular plane systolic excursion, $S P A P$ systolic pulmonary artery pressure, $L V G L S$ left ventricular global longitudinal strain, $C R P C$ reactive protein, $H g b$ Hemoglobin, $R B C$ Red blood cell, $W B C$ White blood cell, Pro- $B N P$ Prohormone B-type natriuretic peptide, $L D H$ Lactate dehydrogenase

*If there is $p<0.05$ as the significance level, $\mathrm{P}^{\mathrm{a}}$ :,control vs without pulmonary involvement $\mathrm{P}^{\mathrm{b}}$ : control vs mild pneumonia, $\mathrm{P}^{\mathrm{c}}$ : control vs severe pneumonia, $\mathrm{P}^{\mathrm{d}}$ : without pulmonary involvement vs mild pneumonia, $\mathrm{P}^{\mathrm{e}}$ : without pulmonary involvement vs severe pneumonia, $\mathrm{P}^{\mathrm{f}}$ : mild pneumonia vs severe pneumonia

close monitoring of long term outcomes such as heart failure and left ventricular dysfunction. Therefore, clinicians should pay close attention to early identification of subclinical myocardial injury by measurements of LVGLS in patients with high serum ferritin and LDH levels after discharge.

To our knowledge, this study is the first to specifically demonstrate the relationship between the biochemical, hematological parameters and LVGLS in patients recovered from COVID-19 in follow-up visits.

COVID-19 infection may present various hematological and biochemical changes according to the severity of inflammatory response. Wan et al. demonstrated that cytokine storm is crucial in the progression from mild to severe disease, leading to ARDS and even death. [16].

Due to the monocyte and macrophage system activation and inflammatory cytokine storm, marked variabilities are observed in inflammatory parameters. Therefore, it is crucial to identify the inflammatory markers associated with the prognosis and outcomes of the disease.

Zeng et al. found that inflammatory markers, particularly CRP, PCT, IL-6 and ferritin were positively correlated with the severity of COVID-19 [17]. In a meta-analysis by Henry et al., in particular, IL-6 and serum ferritin were strong discriminators for severe and fatal COVID-19 disease [18]. Likewise, Qin et al. found CRP, ferritin and LDH higher in severe and critically ill patients than those with non-severe [19]. In another study, Guan et al. presented several biochemical findings so that CRP and elevated LDH showed a more marked increase in more severe cases compared with the non-severe ones [2].

Serum ferritin is a key mediator in immune system by pro-inflammatory effects. It increases in inflammation and contributes to the cytokine storm [20]. Prior studies found serum ferritin levels associated with disease severity and 

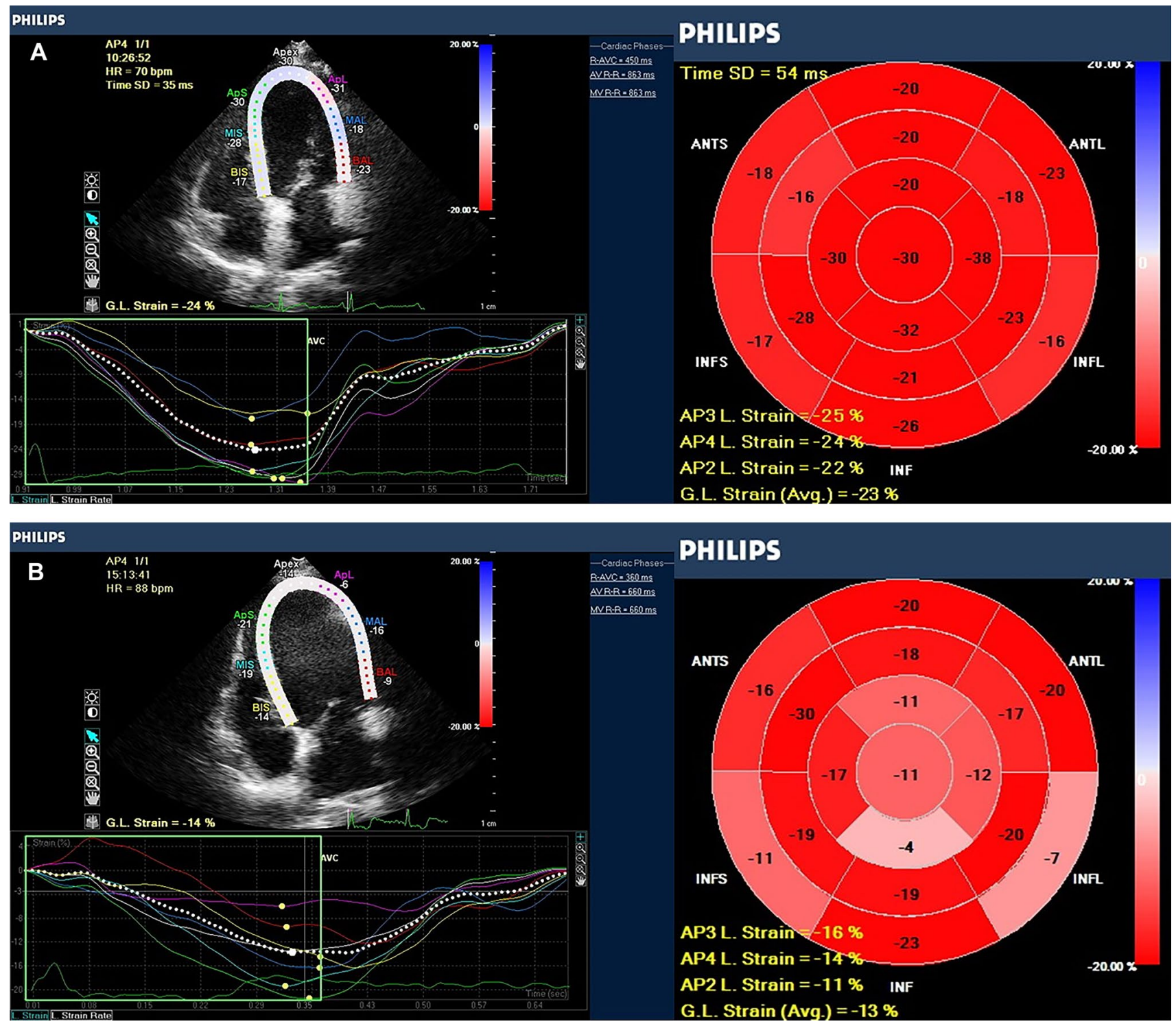

Fig. 2 Bull's eye images of left ventricular global longitudinal strain (LVGLS) of control (a) and study groups (b)

mortality in COVID-19 infection [21, 22]. Besides, Wu et al. found high serum ferritin levels associated with the development of ARDS in COVID-19 [23].

LDH is an intracellular enzyme and is present in cells in almost all organ systems with highest levels in heart, lungs, liver, kidneys, muscles and blood cells. LDH has been used as a marker of acute and chronic tissue damage and is considered an inflammatory marker [24]. Previous studies demonstrated that there was an association between LDH values and worse outcomes in COVID-19 patients. Li et al. found that elevated serum LDH at admission remained a more independent risk factor among the other inflammatory markers for COVID-19 severity and mortality [25]. Therefore, we suggest that elevated serum LDH levels in both acute and chronic phase may be used as an important tool in determining clinical outcomes and prognosis in COVID-19.

In the present study, there was a significant association between the COVID-19 pneumonia and serum ferritin levels both at admission and after discharge. Serum ferritin increased in patients with COVID-19 pneumonia compared to those without pulmonary involvement. Although serum LDH levels in both visits were higher in patients with severe COVID-19 pneumonia, statistical significance was weak. Serum ferritin and LDH levels after discharge were significantly higher in the study group than the control group. CRP, D-dimer, PCT and fibrinogen have been found significant markers in assessing disease severity in many studies, whereas we found no significant association between them in our study. These findings may be related to the small 
Table 3 Comparison of laboratory parameters according to impairment in LVGLS

\begin{tabular}{|c|c|c|c|c|}
\hline & Control $(n=44)$ & Preserved LVGLS $(n=38)$ & Impaired LVGLS $(n=37)$ & $p$-value \\
\hline \multicolumn{5}{|l|}{ Laboratory findings after discharge } \\
\hline Ferritin $(\mathrm{ng} / \mathrm{ml})$ & $32(3-212)^{b}$ & $45.2(7-1010)^{\mathrm{c}}$ & $74.7(14-420.7)^{\mathrm{b}, \mathrm{c}}$ & $0.008^{*}$ \\
\hline Fibrinogen (mg/dl) & $344.5(287-648)$ & $322(180-511)$ & $337(218-472)$ & 0.138 \\
\hline CRP (mg/l) & $2(0-24)$ & $1.3(0.1-18.6)$ & $3.2(0.4-38.8)$ & 0.224 \\
\hline D-dimer $(\mu \mathrm{g} / \mathrm{L})$ & $470(210-1330)$ & $340(180-1910)$ & $320(170-1330)$ & 0.638 \\
\hline $\mathrm{Hgb}(\mathrm{gr} / \mathrm{dl})$ & $13(11-17)^{\mathrm{a}}$ & $12.8(8.5-15.9)^{\mathrm{a}, \mathrm{c}}$ & $13.3(8.8-16.4)^{\mathrm{c}}$ & $0.019^{*}$ \\
\hline $\operatorname{RBC}\left(10^{6} / \mu \mathrm{l}\right)$ & $4.6(3.6-5.7)$ & $4.7(2.8-5.7)$ & $4.8(0.2-5.5)$ & 0.857 \\
\hline Hematocrit (\%) & $40(33.2-49.2)$ & $40(19-47.6)$ & $41(22-48.3)$ & 0.819 \\
\hline Neutrophil/Lymphocyte Ratio & $1.9(0.6-3.8)$ & $1.9(0.6-5.5)$ & $1.6(0.7-7.6)$ & 0.723 \\
\hline Neutrophil $\left(10^{3} / \mu \mathrm{l}\right)$ & $3.900(1.6-7.8)$ & $3.4(2.4-8)$ & $3.6(1.4-16.6)$ & 0.446 \\
\hline Lymphocyte $\left(10^{3} / \mu \mathrm{l}\right)$ & $2.4(1.1-6.9)$ & $1.900(0.8-4.1)$ & $2.200(0.5-4.1)$ & 0.203 \\
\hline $\operatorname{WBC}\left(10^{3} / \mu \mathrm{l}\right)$ & $7(4.4-13.5)$ & $6(3.4-12)$ & $6.6(2.4-19.6)$ & 0.302 \\
\hline Lymphocyte \% & $33.2 \pm 7.8$ & $32.9 \pm 6.7$ & $32.4 \pm 8.7$ & 0.911 \\
\hline Neutrophil \% & $57.1 \pm 7.9$ & $57 \pm 7$ & $56.9 \pm 9.6$ & 0.992 \\
\hline Platelet $\left(10^{3} / \mu \mathrm{l}\right)$ & $241(179-373)^{\mathrm{b}}$ & $274(39.6-409)^{c}$ & $214.5(134-327)^{\mathrm{b}, \mathrm{c}}$ & $<0.001^{*}$ \\
\hline Pro-BNP (pg/ml) & $34(4-44)^{\mathrm{a}, \mathrm{b}}$ & $54.8(5-1258)^{\mathrm{a}}$ & $66.5(5-621.5)^{\mathrm{b}}$ & $0.017^{*}$ \\
\hline $\operatorname{AST}(\mathrm{U} / \mathrm{l})$ & $17.6(9.3-82.9)$ & $18.8(12-237)$ & $20.1(10.4-51.1)$ & 0.255 \\
\hline $\operatorname{ALT}(\mathrm{U} / \mathrm{l})$ & $18.2(8-80.3)$ & $18(5.9-205)$ & $23.7(4.9-77.7)$ & 0.827 \\
\hline $\operatorname{BUN}(\mathrm{mg} / \mathrm{dl})$ & $11.9(5.9-44.3)$ & $13.5(6-20.5)$ & $14.1(7.7-37.1)$ & 0.428 \\
\hline Creatinine $(\mathrm{mg} / \mathrm{dl})$ & $0.8(0.5-1.2)$ & $0.8(0.4-1.7)$ & $0.8(0.6-1.4)$ & 0.547 \\
\hline $\mathrm{LDH}(\mathrm{U} / \mathrm{l})$ & $176.3 \pm 47.3^{\mathrm{b}}$ & $183.2 \pm 29.8^{\mathrm{c}}$ & $208 \pm 34.4^{\mathrm{b}, \mathrm{c}}$ & $0.001^{*}$ \\
\hline Uric acid $(\mathrm{mg} / \mathrm{dl})$ & $4.9 \pm 1.6$ & $5.2 \pm 1.7$ & $5.4 \pm 1.6$ & 0.317 \\
\hline Fasting Plasma Glucose (mg/dl) & $92.5(69-159)$ & $89.5(69-165)$ & $95(76-268)$ & 0.157 \\
\hline \multicolumn{5}{|c|}{ Laboratory findings at hospital admission } \\
\hline Ferritin (ng/ml) & - & $216.2(28.3-1718)$ & $278.6(22-1654)$ & 0.148 \\
\hline Fibrinogen $(\mathrm{mg} / \mathrm{dl})$ & - & $479.5(301-714)$ & $473(204-747)$ & 0.857 \\
\hline $\mathrm{CRP}(\mathrm{mg} / \mathrm{l})$ & - & $17.6(0.7-95.1)$ & $30.3(1.5-230.2)$ & 0.180 \\
\hline Procalcitonin (ng/ml) & - & $0.1(0-29.6)$ & $0.1(0-77.7)$ & 0.607 \\
\hline $\mathrm{D}$-dimer $(\mu \mathrm{g} / \mathrm{L})$ & - & $655(270-3440)$ & $740(280-18.550)$ & 0.394 \\
\hline $\mathrm{Hgb}(\mathrm{gr} / \mathrm{dl})$ & - & $13.1(9.7-16.1)$ & $13(8.1-16.8)$ & 0.830 \\
\hline $\operatorname{RBC}\left(10^{6} / \mu \mathrm{l}\right)$ & - & $4.4(3-486)$ & $4.7(2.9-5.8)$ & 0.339 \\
\hline Hematocrit (\%) & - & $38.3(26.5-48.3)$ & $39.7(24.6-50.5)$ & 0.301 \\
\hline Neutrophil/Lymphocyte Ratio & - & $3.3(1-43.2)$ & $2.6(1.2-43.5)$ & 0.495 \\
\hline Neutrophil $\left(10^{3} / \mu \mathrm{l}\right)$ & - & $3.5(1.9-9.7)$ & $3.4(1.6-11.3)$ & 0.684 \\
\hline Lymphocyte $\left(10^{3} / \mu \mathrm{l}\right)$ & - & $1.3(0.8-5)$ & $1.5(0.200-3)$ & 0.588 \\
\hline $\operatorname{WBC}\left(10^{3} / \mu \mathrm{l}\right)$ & - & $6.2(3.2-15.9)$ & $5.2(3.3-12)$ & 0.687 \\
\hline Lymphocyte \% & - & $24.1 \pm 9.8$ & $161 \pm 643.4$ & 0.303 \\
\hline Neutrophil \% & - & $66.6 \pm 10.5$ & $65.3 \pm 12.1$ & 0.692 \\
\hline Platelet $\left(10^{3} / \mu \mathrm{l}\right)$ & - & $223.5(118.5-1100)$ & $182(73.7-344.1)$ & 0.218 \\
\hline Pro-BNP (pg/ml) & - & $46.9(8.5-415.6)$ & $83.4(7.4-793.1)$ & 0.141 \\
\hline $\operatorname{AST}(\mathrm{U} / \mathrm{l})$ & - & $27.3(14-45.9)$ & $29.9(7.9-76.4)$ & 0.451 \\
\hline ALT (U/l) & - & $24.8(9.6-66)$ & $25.5(7.8-276)$ & 0.914 \\
\hline BUN (mg/dl) & - & $11.7(5.2-30.8)$ & $13.7(4.6-36)$ & 0.123 \\
\hline Creatinine $(\mathrm{mg} / \mathrm{dl})$ & - & $0.8(0.3-2)$ & $0.8(0.5-1.5)$ & 0.683 \\
\hline $\mathrm{LDH}(\mathrm{U} / \mathrm{l})$ & - & $275.9 \pm 118.5$ & $320.9 \pm 147.3$ & 0.371 \\
\hline Uric acid $(\mathrm{mg} / \mathrm{dl})$ & - & $4.3 \pm 1.4$ & $5.3 \pm 2.3$ & 0.168 \\
\hline Fasting Plasma Glucose (mg/dl) & - & $104(90-135.2)$ & $114.7(84.6-242)$ & 1.000 \\
\hline
\end{tabular}

Abbreviations: $C R P$ C reactive protein, $H g b$ hemoglobin, $R B C$ red blood cell, $W B C$ white blood cell, Pro- $B N P$ prohormone B-type natriuretic peptide, $A S T$ aspartate transaminase, $A L T$ alanine transaminase, $B U N$ blood urea nitrogen, $L D H$ lactate dehydrogenase, $L V G L S$ left ventricular global longitudinal strain

*If there is $p<0.05$ as the significance level, $\mathrm{P}^{\mathrm{a}}$ : control vs preserved LVGLS, $\mathrm{P}^{\mathrm{b}}$ : control vs impaired LVGLS, $\mathrm{P}^{\mathrm{c}}$ : preserved LVGLS vs impaired LVGLS 

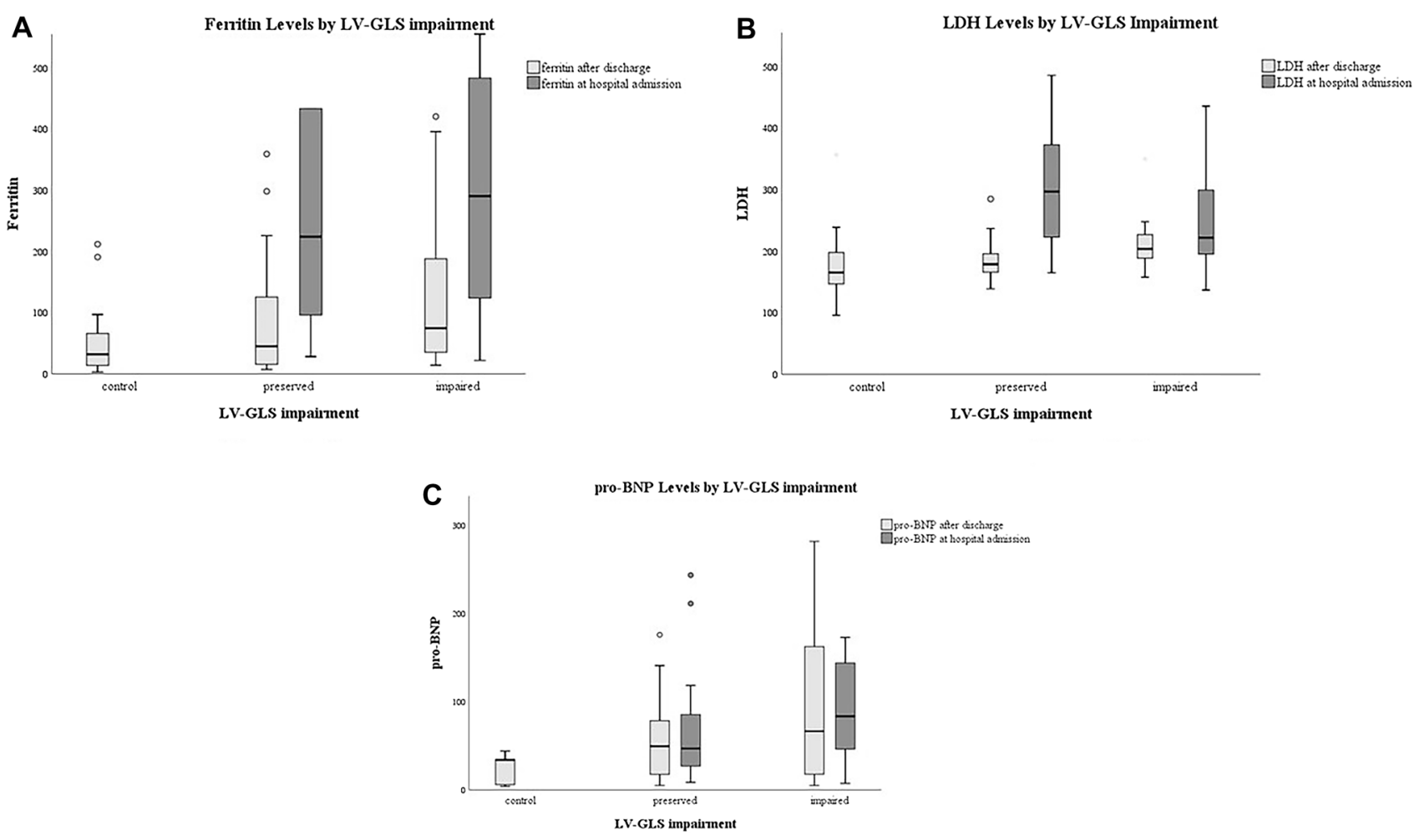

Fig. 3 a Serum Ferritin levels according to left ventricular global longitudinal strain (LVGLS) impairment of the study group. b Serum LDH levels according to LVGLS impairment of the study group. $\mathbf{c}$ Serum pro-BNP levels according to LVGLS impairment of the study group

Table 4 Correlation of LVGLS with inflammatory markers both at hospital admission and after discharge. a: Correlation of LVGLS with age, pneumonia severity and inflammatory markers at hospital admission. b: Correlation of LVGLS with inflammatory markers after discharge

\begin{tabular}{|c|c|c|c|c|c|c|c|c|c|c|c|c|c|}
\hline (a) & Spearman & Age & Pneun & ia Severity & Ferritin & CRP & Fibrinogen & $\mathrm{Hgb}$ & Lymph & ocyte & PCT & Neutrophil & NLR \\
\hline \multirow[t]{2}{*}{ LVGLS (\%) } & $\mathrm{R}$ & -0.270 & -0.35 & & -0.300 & -0.280 & -0.016 & 0.045 & 0.090 & & 0.007 & -0.083 & -0.170 \\
\hline & $P$ & $0.004^{*}$ & $<0.0$ & & $0.010^{*}$ & $0.010^{*}$ & 0.912 & 0.697 & 0.429 & & 0.959 & 0.453 & 0.147 \\
\hline (b) & \multicolumn{2}{|c|}{ Spearman } & Ferritin & CRP & \multicolumn{2}{|c|}{ Fibrinogen } & $\mathrm{Hgb}$ & \multicolumn{2}{|c|}{ Lymphocyte } & \multicolumn{2}{|c|}{ Neutrophil } & NLR & $\mathrm{LDH}$ \\
\hline \multirow[t]{2}{*}{ LVGLS (\%) } & \multicolumn{2}{|l|}{$\mathrm{R}$} & -0.252 & -0.169 & \multicolumn{2}{|c|}{-0.218} & -0.191 & \multicolumn{2}{|l|}{-0.130} & \multicolumn{2}{|c|}{-0.141} & 0.013 & -0.268 \\
\hline & \multicolumn{2}{|l|}{$\mathrm{P}$} & $0.012^{*}$ & 0.077 & \multicolumn{2}{|l|}{0.053} & $0.044^{*}$ & \multicolumn{2}{|l|}{0.171} & \multicolumn{2}{|c|}{0.138} & 0.897 & $0.005^{*}$ \\
\hline
\end{tabular}

Abbreviations: $C R P$ C reactive protein, $H g b$ hemoglobin, $L D H$ lactate dehydrogenase, $P C T$ procalcitonin, $N L R$ Neutrophil-to-Lymphocyte ratio, $L V G L S$ left ventricular global longitudinal strain

sample size, especially in the severe pneumonia group. We suggested that serum ferritin may be a more sensitive and potent inflammatory marker in the prediction of the disease severity among the others in both acute and chronic stages of the disease.

Neutrophilia and lymphocytopenia occur as physiological responses of the innate immune system to systemic inflammation. In COVID-19 disease, inflammatory cytokines may accelerate lymphocyte apoptosis [26] and further trigger the migration of blood lymphocytes to lymph nodes and into inflamed tissues; leading to inflammatory lymphopenia. Recently, NLR, a composite index of systemic inflammation has been proposed as a novel prognostic marker in both cardiac and non-cardiac diseases [27, 28]. In many studies, NLR was found to be significantly higher in severe COVID19 disease [29]. NLR has been suggested as an independent risk factor for predicting COVID-19 infection and it may determine the prognosis [29].

However, our study demonstrated no statistical difference between NLR and pneumonia severity in both visits. On the other hand, lymphocyte and WBC counts slightly increased in patients with severe pneumonia in follow-up visits. This was an interesting finding in the present study and this may potentially suggest that the increase in WBC and lymphocyte 

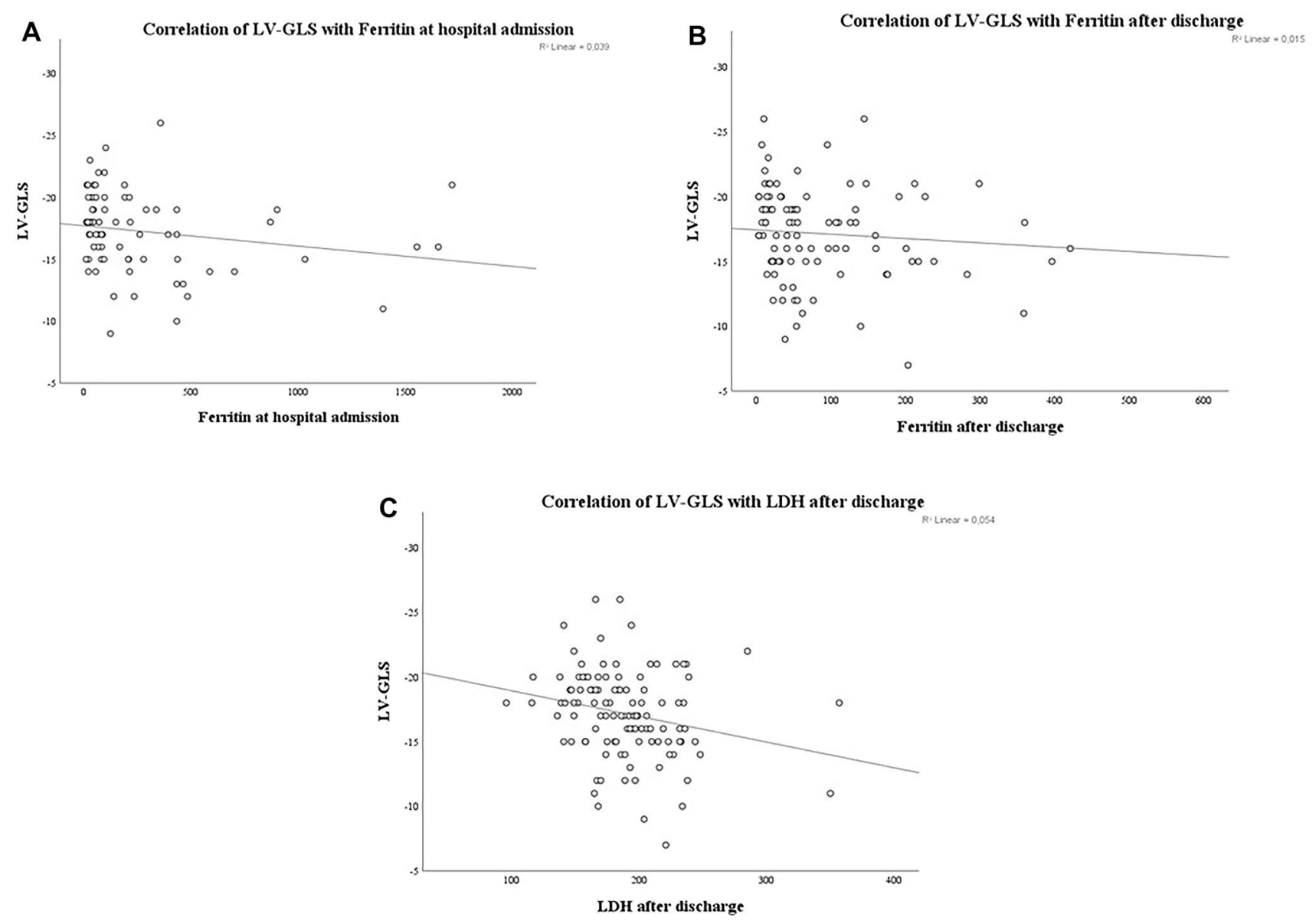

Fig. 4 Correlation of LVGLS with Ferritin at hospital admission (a) and after discharge (b). Correlation of LVGLS with LDH after discharge (c)

counts may have occurred as a compensatory response to systemic inflammatory changes in the chronic phase.

COVID-19 disease can affect multiple organ systems, however the higher mortality in patients with COVID-19 may be attributed to cardiovascular involvement with worsening prognosis. The mechanisms of cardiovascular injury may be due to multiple factors.

Myocardial injury may occur through direct and indirect mechanisms in COVID-19 patients. Direct cardiotoxicity may occur by viral infiltration of myocardium as direct mechanism. Indirect mechanisms may involve stress-induced cardiomyopathy through respiratory failure and hypoxemia and myocardial inflammation by pro-inflammatory cytokines [30]. Activation of the immune response with cytokine storm leads to myocardial inflammation and myocardial suppression [31]. Morever, hypercytokinemia causes endothelial damage, generating a state of hypercoagulability, plaque instability and thromboembolic events [31, 32]. However, the main components of the myocardial injury are inflammatory mechanisms in COVID-19 [30].

The early identification of myocardial injury is crucial to prevent poor clinic outcomes and mortality [33]. LVGLS analysis by STE demonstrates myocardial deformation earlier and may determine subclinical left ventricular myocardial dysfunction in case of preserved LVEF.

Recently, GLS analysis using STE is a sensitive and reliable method for determining occult myocardial injury and it has additional prognostic information [34, 35]. Longitudinal myocardial fibres are located in the subendocardium which is most vulnerable to ischemia or injury [36]. Therefore, GLS may identify myocardial damage at an early stage and prior to reduction in LVEF. The identification of subclinical LV dysfunction by GLS is important in many clinical conditions. For instance, in patients undergoing chemotherapy GLS is recommended in addition to LVEF to detect subclinical systolic dysfunction and prevent overt heart failure [37]. Furthermore, LVGLS imaging may be used to diagnose myocardial ischaemia and in patients with valvular heart disease, it may reflect myocardial function prior to fall in LVEF. Reduction in LVEF may imply irreversible myocardial damage and it may be too late for medical therapy [38]. Besides, it may be associated with increased risk of ventricular arrhythmias. However, GLS imaging showing early LV systolic dysfunction may provide a new perspective and 
A

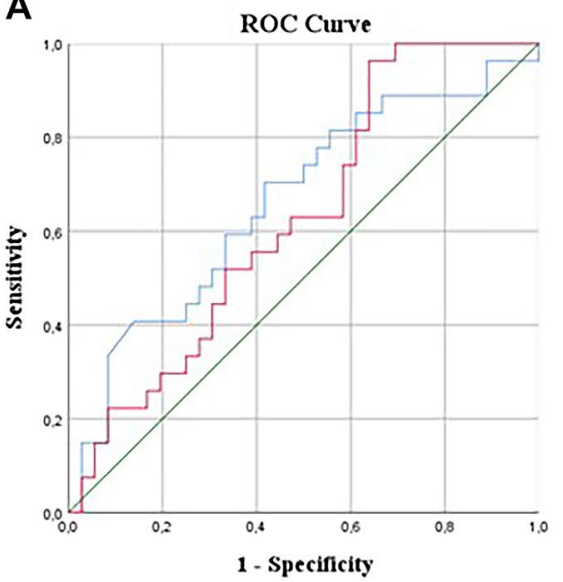

Source of the Curve - Eemion at hospital admission - Eemon ater dascharge

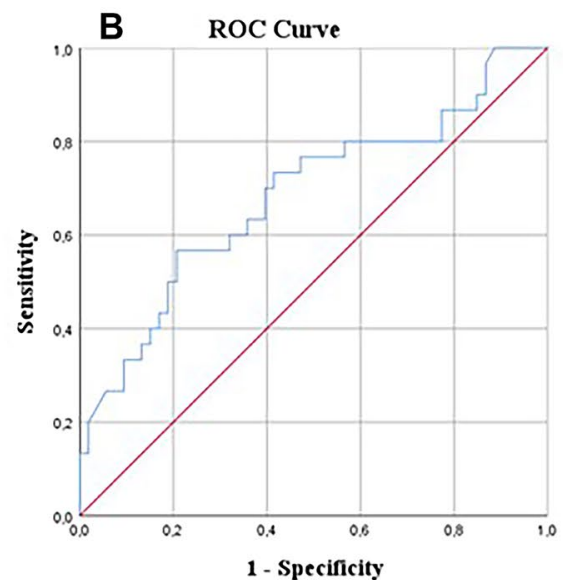

CRP at hospital admission

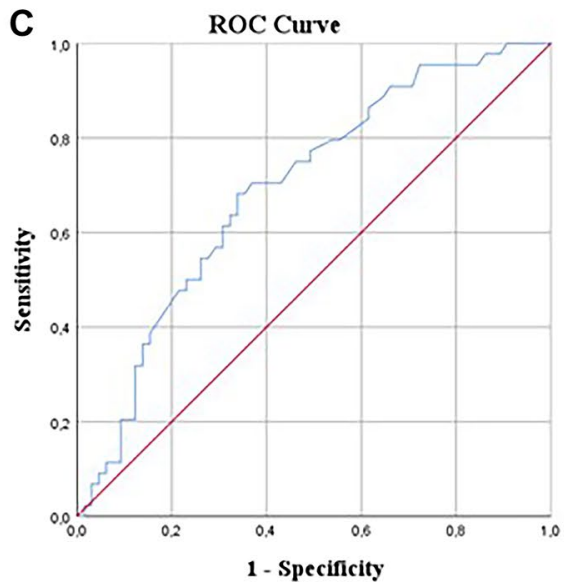

LDH atter dischare

Fig. 5 a ROC curve analysis for predicting impaired LVGLS by Ferritin both at hospital admission and after discharge. b ROC curve analysis for predicting impaired LVGLS by CRP at hospital admission. $\mathbf{c}$ ROC curve analysis for predicting impaired LVGLS by LDH after discharge

prevention with therapeutic approaches and lifestyle changes in heart failure prophylaxis.

Medical therapy based on standard heart failure guideline with beta-blockers and angiotensin-converting enzyme inhibitörs (ACEI) may be treatment options to prevent LV dysfunction as any other heart failure patient [39]. For this reason, in the follow-up period, to measure GLS in addition to LVEF will be helpful in cases recovering from COVID19 even though LVEF is in the normal range. COVID-19 patients with impaired LVGLS values will benefit from closer monitoring of cardiac function.

The present study showed that serum ferritin, LDH and pro-BNP values after discharge were significantly higher in COVID-19 patients with impaired LVGLS. There was a significant correlation between serum ferritin, LDH levels and LVGLS at a median 6 months after discharge. Serum ferritin and LDH levels were negatively correlated with LVGLS values in patients recovered from COVID-19 in follow up visits. We found no statistical difference between LVGLS and the other inflammatory markers both at admission and after discharge. Additionally, we found higher hemoglobin values and lower platelet counts in patients with impaired LVGLS than those with preserved. These results may be related to the outcomes of the severe COVID-19 disease in the chronic settings.

Herein, we may deduce that patients- once recovered from the COVID-19 infection with higher serum ferritin and LDH levels after discharge should be observed closely for future cardiac events such as left ventricular dysfunction, heart failure or arrhythmia in long term follow-up.

\section{Limitations of the study}

This study has several limitations. First, the sample size of the study groups was relatively small since it is a single center study. Second, we used inflammatory markers such 
as ferritin, CRP, PCT, NLR, D-Dimer and LDH levels to represent inflammation. However, it would be better to measure interleukins such as IL-6, IL-1, and TNF-alpha which play an important role in inflammatory response in COVID-19 to have more validated results. Third, we have no endpoints such as mortality, or prognosis of patients affected by COVID-19. Long term follow-up is needed to show the impact of reduced LVGLS for future cardiac events.

\section{Conclusion}

In this study, inexpensive and easily available clinic parameters/inflammatory markers are investigated for predicting the severity of COVID-19 pneumonia, occult myocardial injury and prognosis in follow-up. In conclusion, serum ferritin and LDH values are found to be statistically significant and would provide important clues for clinicians to pay close attention to the management of the disease and the early identification of left ventricular dysfunction and myocardial injury in follow up visits. Further studies with larger sample sizes are needed to confirm our results.

Acknowledgements We thank Yunus Govdeli for English grammar editing.

Funding None.

\section{Declarations}

Conflict of interest The author(s) declared no potential conflicts of interest concerning the research, authorship, and/or publication of this article.

\section{References}

1. Arshad Ali S, Baloch M, Ahmed N et al (2020) The outbreak of coronavirus disease 2019 (COVID-19)-an emerging global health threat. J Infect Public Health 13:644-646. https://doi.org/ 10.1016/j.jiph.2020.02.033

2. Guan WJ, Ni ZY, Hu Y et al (2020) Clinical characteristics of coronavirus disease 2019 in China. N Engl J Med 382:1708-1720. https://doi.org/10.1056/NEJMoa2002032

3. Zhou Y, Fu B, Zheng X et al (2020) Pathogenic T cells and inflammatory monocytes incite inflammatory storm in severe COVID-19 patients. Natl Sci Rev 13:nwaa041. https://doi.org/10.1093/nsr/ nwaa041

4. Cheng K, Wei M, Shen $\mathrm{H}$ et al (2020) Clinical characteristics of 463 patients with common and severe type coronavirus disease (In Chinese). Shanghai Med J 1-15.

5. Gao Y, Li T, Han M et al (2020) Diagnostic utility of clinical laboratory data determinations for patients with the severe COVID-19. J Med Virol 92:791-796

6. Chan JF, Yuan S, Kok KH et al (2020) A familial cluster of pneumonia associated with the 2019 novel coronavirus indicating person-to-person transmission: a study of a family cluster. Lancet 395:514-523. https://doi.org/10.1016/S0140-6736(20)30154-9
7. Guo T, Fan Y, Chen M et al (2020) Cardiovascular implications of fatal outcomes of patients with coronavirus disease 2019 (COVID-19). JAMA Cardiol. https://doi.org/10.1001/jamacardio. 2020.1017

8. Zheng YY, Ma YT, Zhang JY et al (2020) COVID-19 and the cardiovascular system. Nat Rev Cardiol 17:259-260

9. Biering-Sorensen T, Biering-Sorensen SR, Olsen FJ et al (2017) Global longitudinal strain by echocardiography predicts long-term risk of cardiovascular morbidity and mortality in a low risk general population: the Copenhagen City Heart study. Circ Cardiovasc Imaging 10(3):e005521

10. Ersboll M, Valeur N, Mogensen UM et al (2013) Prediction of all-cause mortality and heart failure admissions from global left ventricular longitudinal strain in patients with acute myocardial infarction and preserved left ventricular ejection fraction. J Am Coll Cardiol 61:2365-2373

11. Thavendiranathan P, Poulin F, Lim KD et al (2014) Use of myocardial strain imaging by echocardiography for the early detection of cardiotoxicity in patients during and after cancer chemotherapy: a systematic review. J Am Coll Cardiol 63:2751-2768

12. Lang RM, Bierig M, Devereux RB et al (2005) Recommendations for chamber quantification: a report from the American Society of Echocardiography's Guidelines and Standards Committee and the Chamber Quantification Writing Group, developed in conjunction with the European Association of Echocardiography, a branch of the European Society of Cardiology. J Am Soc Echocardiogr 18:1440-1463

13. Schiller NB, Acquatella H, Ports TA et al (1979) Left ventricular volume from paired biplane two dimensional echocardiography. Circulation 60:547-555

14. Yingchoncharoen T, Agarwal S, Popovic ZB et al (2013) Normal ranges of left ventricular strain: a meta-analysis. J Am Soc Echocardiogr 26:185-191

15. Yang H, Wright L, Negishi T et al (2018) Research to practice: assessment of left ventricular global longitudinal strain for surveillance of cancer chemotherapeutic-related cardiac dysfunction. JACC Cardiovasc Imaging 11:1196-1201

16. Wan SX, Yi QJ, Fan SB et al (2020) Characteristics of lymphocyte subsets and cytokines in peripheral blood of 123 hospitalized patients with 2019 novel coronavirus pneumonia (NCP). meDrxiv. https://doi.org/10.1101/2020.02.10.20021832

17. Zeng F, Huang Y, Guo Y et al (2020) Association of inflammatory markers with the severity of COVID-19: a meta-analysis. Int J Infect Dis 96:467-474

18. Henry BM, de Oliveira MHS, Benoit S et al (2020) Hematologic, biochemical and immune biomarker abnormalities associated with severe illness and mortality in coronavirus disease 2019 (COVID-19): a meta-analysis. Clin Chem Lab Med. https://doi. org/10.1515/cclm-2020-0369

19. Qin C, Zhou L, Hu Z et al (2020) Dysregulation of immune response in patients with COVID-19 in Wuhan, China. Clin Infect Dis 2020;.

20. Abbaspour N, Hurrell R, Kelishadi R (2014) Review on iron and its importance for human health. Research J Med Sci 19:164-174

21. Tural Onur S, Altın S, Sokucu SN et al (2020) Could ferritin level be an indicator of COVID-19 disease mortality? J Med Virol. https://doi.org/10.1002/jmv.26543

22. Liu T, Zhang J, Yang Y et al (2020) The potential role of IL-6 in monitoring severe case of coronavirus disease 2019. meDrxiv. https://doi.org/10.1101/2020.03.01.20029769

23. Wu C, Chen X, Cai Y et al (2020) Risk factors associated with acute respiratory distress syndrome and death in patients with coronavirus disease 2019 pneumonia in Wuhan, China. JAMA Intern Med. https://doi.org/10.1001/jamainternmed.2020.0994

24. J Sepulveda (2013) Challenges in Routine Clinical Chemistry Analysis: Proteins and Enzymes. Editor(s): A. Dasgupta, J. L. 
Sepulveda, Chapter 9, Accurate Results in the Clinical Laboratory. Elsevier 131-148.

25. Li C, Ye J, Chen Q et al (2020) Elevated Lactate Dehydrogenase $(\mathrm{LDH})$ level as an independent risk factor for the severity and mortality of COVID-19. Aging 12:15670-15681

26. Chan PK, Chen GG (2008) Mechanisms of lymphocyte loss in SARS coronavirus infection. Hong Kong Med J 14:21-26

27. Balta S, Demirkol S, Unlu M et al (2013) Neutrophil to lymphocyte ratio may be predict of mortality in all conditions. Br J Cancer 109:3125

28. IJsselmuiden AJ, Musters RJ, De Ruiter G et al (2008) Circulating white blood cells and platelets amplify oxidative stress in heart failure. Nat Rev Cardiol 5:811

29. Yufei Y, Mingli L, Xuejiao L et al (2020) Utility of the neutrophilto-lymphocyte ratio and C-reactive protein level for coronavirus disease 2019 (COVID-19). Scand J Clin Lab Invest 80:536-540

30. Akbarshakh A, Marban E (2020) COVID-19 and the heart. Circ Res 126:1443-1455

31. Prabhu SD (2004) Cytokine-induced modulation of cardiac function. Circ Res 95:1140-1153

32. Levi M, van der Poll T, Büller HR (2004) Bidirectional relation between inflammation and coagulation. Circulation 109:2698-2704

33. Zhou B, She J, Wang Y et al (2020) The clinical characteristics of myocardial injury in severe and very severe patients with novel coronavirus disease. J Infect. https://doi.org/10.1016/j.jinf.2020. 03.021

34. Potter E, Marwick TH (2018) Assessment of left ventricular function by echocardiography: the case for routinely adding global longitudinal strain to ejection fraction. JACC Cardiovasc Imaging 11:260-274

35. Jung IH, Park JH, Lee JA et al (2020) Left ventricular global longitudinal strain as a predictor for left ventricular reverse remodeling in dilated cardiomyopathy. J Cardiovasc Imaging 28:137-149

36. Kalam K, Otahal P, Marwick TH (2014) Prognostic implications of global LV dysfunction: a systematic review and metaanalysis of global longitudinal strain and ejection fraction. Heart 100:1673-1680

37. Plana JC, Galderisi M, Barac A et al (2014) Expert consensus for multimodality imaging evaluation of adult patients during and after cancer therapy: a report from the American Society of Echocardiography and the European Association of Cardiovascular Imaging. Eur Heart J Cardiovasc Imaging 15:1063-1093

38. Cardinale D, Colombo A, Lamantia G et al (2010) Anthracyclineinduced cardiomyopathy: clinical relevance and response to pharmacologic therapy. J Am Coll Cardiol 55:213-220

39. Negishi K, Negishi T, Haluska BA et al (2014) Use of speckle strain to assess left ventricular responses to cardiotoxic chemotherapy and cardioprotection. Eur Heart J Cardiovasc Imaging $15: 324-331$

Publisher's Note Springer Nature remains neutral with regard to jurisdictional claims in published maps and institutional affiliations. 